\title{
Long-term Follow-up of a Randomized Controlled Trial of Suture Versus Mesh Repair of Incisional Hernia
}

\author{
Jacobus W. A. Burger, MD, * Roland W. Luijendijk, PhD, † Wim C. J. Hop, PhD, f \\ Jens A. Halm, MD, * Emiel G. G. Verdaasdonk, MD, * and Johannes Jeekel, PhD*
}

\begin{abstract}
Objective: The objective of this study was to determine the best treatment of incisional hernia, taking into account recurrence, complications, discomfort, cosmetic result, and patient satisfaction.

Background: Long-term results of incisional hernia repair are lacking. Retrospective studies and the midterm results of this study indicate that mesh repair is superior to suture repair. However, many surgeons are still performing suture repair.
\end{abstract}

Methods: Between 1992 and 1998, a multicenter trial was performed, in which 181 eligible patients with a primary or first-time recurrent midline incisional hernia were randomly assigned to suture or mesh repair. In 2003, follow-up was updated.

Results: Median follow-up was 75 months for suture repair and 81 months for mesh repair patients. The 10-year cumulative rate of recurrence was $63 \%$ for suture repair and $32 \%$ for mesh repair $(P<$ $0.001)$. Abdominal aneurysm $(P=0.01)$ and wound infection $(P=$ $0.02)$ were identified as independent risk factors for recurrence. In patients with small incisional hernias, the recurrence rates were $67 \%$ after suture repair and $17 \%$ after mesh repair $(P=0.003)$. One hundred twenty-six patients completed long-term follow-up (median follow-up 98 months). In the mesh repair group, $17 \%$ suffered a complication, compared with $8 \%$ in the suture repair group $(P=0.17)$. Abdominal pain was more frequent in suture repair patients $(P=0.01)$, but there was no difference in scar pain, cosmetic result, and patient satisfaction.

Conclusions: Mesh repair results in a lower recurrence rate and less abdominal pain and does not result in more complications than suture repair. Suture repair of incisional hernia should be abandoned.

(Ann Surg 2004;240: 578-585)

From the *Department of General Surgery, Erasmus University Medical Center, Rotterdam, the Netherlands; $†$ Department of Plastic and Reconstructive Surgery, Antonius Ziekenhuis, Nieuwegein, The Netherlands; and $\ddagger$ Department of Epidemiology and Biostatistics, Erasmus University Medical Center, Rotterdam, The Netherlands.

Reprints: J. Jeekel, Department of General Surgery, Erasmus University Medical Center, P.O. Box 2040, 3000 CA Rotterdam, The Netherlands. E-mail: j.jeekel@erasmusmc.nl.

Copyright (C) 2004 by Lippincott Williams \& Wilkins

ISSN: 0003-4932/04/24004-0578

DOI: $10.1097 / 01$. sla.0000141193.08524.e7 ncisional hernia remains a frequent complication of abdominal surgery, with a reported incidence of $2 \%$ to $20 \% .{ }^{1-5} \mathrm{In}$ the United States, 4 to 5 million laparotomies are performed annually, ${ }^{6}$ which means that at least 400,000 to 500,000 incisional hernias can be expected to develop each year. Incisional hernia repair is performed approximately 200,000 times per year. ${ }^{6,7}$ In the Netherlands, 100,000 laparotomies and 3900 incisional hernia repairs are performed annually (data obtained from Prismant ${ }^{8}$ ). These data imply that in both countries, and probably in general, $4 \%$ of patients undergoing a laparotomy will go through additional surgery to repair an incisional hernia. When morbidity is added to the vast numbers and the tremendous costs associated with incisional hernia repair, ${ }^{9}$ it becomes clear that the efficacy of incisional hernia repair is of major importance. Unfortunately, results of incisional hernia repair are disappointing. Suture repair of incisional hernia results in recurrence rates of $12 \%$ to $54 \%,{ }^{10-16}$ while mesh repair results in recurrence rates of $2 \%$ to $36 \%{ }^{13-15,17-23}$ Because most studies only provide shortterm follow-up, these recurrence rates may even be underestimated. In addition to the high recurrence rates, incisional hernia repair may give rise to serious complications, such as enterocutaneous fistula and bowel obstruction, causing deterioration rather than improvement of the patient's situation. Furthermore, patients may suffer pain, and the cosmetic result of incisional hernia repair may be disappointing.

In 2000, a randomized controlled trial by our group indicated that mesh repair is superior to suture repair, even for small incisional hernias. ${ }^{14}$ Unfortunately, there have not been randomized trials of incisional hernia repair since, and some authors have even suggested that there may still be a place for suture repair of incisional hernia. ${ }^{15,24,25}$ Disconcerting data indicate that surgeons are still performing suture repair, in spite of clinical evidence. In 1997, in Germany, $85 \%$ of incisional hernias repairs was still performed without prosthetic mesh, ${ }^{26}$ while in 1999, in Washington state, $35 \%$ of incisional hernias was repaired without mesh. ${ }^{27}$ In the Netherlands in 2002, surgeons failed to use a mesh in $40 \%$ of incisional hernia repairs. ${ }^{8}$ 
To determine what type of hernia repair should be performed, long-term results of incisional hernia repair are needed. The purpose of this study was to provide these long-term results. Patients who participated in a randomized controlled trial on incisional hernia repair, which included 181 patients between 1992 and 1998, were asked to complete a questionnaire and visit the outpatient clinic.

\section{METHODS}

Between March 1992 and February 1998, we randomly assigned 200 adult patients with a primary or first recurrent incisional hernia to suture repair or mesh repair.

Singular small $(\leq 6 \mathrm{~cm})$ midline incisional hernias were included. Patient-related factors that were recorded were gender, age, obesity, cough, constipation, prostatism, diabetes mellitus, glucocorticoid therapy, smoking status, and abdominal surgical history. Operation related factors that were recorded were surgical technique, size of the defect, presence of hematoma, dehiscence, and wound infection. During suture repair, the edges of the fascia were approximated in the midline with a continuous polypropylene suture (Prolene no. 1; Ethicon, Amersfoort, the Netherlands). In patients assigned to mesh repair, a polypropylene mesh (Prolene, Ethicon or Marlex, Bard Benelux, Nieuwegein, the Netherlands) was tailored to the defect so that at least $2 \mathrm{~cm}$ of the mesh overlapped the fascia, and the mesh was sutured to the back of the abdominal wall with a continuous polypropylene suture. Any peritoneal defect was closed or the omentum was sutured between bowels and mesh. When this could not be done, a polyglactin 910 (Vicryl; Ethicon) mesh was fixed in between. The study was approved by the ethics committees of the participating hospitals, and all patients gave informed consent. A more meticulous description of inclusion and exclusion criteria, surgical technique, and recorded patient characteristics has been published previously. ${ }^{14}$

In 2003, all patients were asked to complete a questionnaire. Patients were asked whether they had suffered a recurrence, scar pain, abdominal pain, mesh infection, incarcerated hernia, small bowel obstruction, or enterocutaneous fistula. Patients were also asked whether they had undergone hernia repair since their last visit, and patients were asked to score pain in a visual analogue scale. They were asked to rate the cosmetic appearance of their abdomen and to state whether they were ashamed of the appearance of the abdomen. Finally, patients were asked whether they were satisfied with the result of the operation. Patients were invited to visit the outpatient clinic, where a patient history was taken and a physical examination performed. The abdomen was examined for hernia recurrence, which was defined as any fascial defect that was palpable or detected by ultrasound examination and was located within $7 \mathrm{~cm}$ of the site of hernia repair. The examination included palpation while the patient was in the supine position with legs extended and raised. Ultrasound examination was performed when physical examination was not conclusive.

\section{Statistical Analysis}

Percentages and continuous variables were compared with the use of Fisher exact test and the Mann-Whitney test, respectively. The cumulative percentages of patients with recurrences over time were calculated and compared with use of Kaplan-Meier curves and log-rank tests. Multivariate analysis of various factors was performed with Cox regression analysis. Through the use of appropriate interaction terms, we investigated whether the effect of treatment depended on the size of the repaired hernia. All statistical tests were 2-sided. The primary analysis was performed on an intention-to-treat basis; that is, patients remained in their assigned group even if during the procedure the surgeon judged the patient not to be suitable for the technique assigned.

\section{RESULTS}

Among the 200 patients enrolled in the study, 171 had a primary incisional hernia and 29 had a first recurrence of incisional hernia. Nineteen patients were found to be ineligible for the study for reasons described previously. ${ }^{14}$ At baseline, there were no significant differences between patients in the suture repair and the mesh repair groups (Table 1). Ninety-seven patients were assigned to suture repair and 84 to mesh repair. Seven patients assigned to the suture repair group underwent mesh repair and 5 patients assigned to the mesh-repair group underwent suture repair.

During the 2003 update, 126 patients were successfully contacted. Thirty-nine patients had died, 11 patients could not be located, and 5 patients refused to cooperate. Causes of death were not related to the repair but were cardiovascular (12), cerebrovascular (6), malignancy (10), per operative (not incisional hernia repair related; 5), pulmonary (5), and alcohol and drug-abuse related (1). Inability to locate patients was due to patients having moved (5) and emigrated (6). Reasons for refusing cooperation were: not wanting to take leave from work (2), lack of interest (1) and unknown (2). One hundred twenty-six of 142 living patients $(89 \%)$ were seen at the outpatient clinic. Data of the 55 patients that did not visit the outpatient clinic during the current update were censored to their previous visit. With data of the 2003 update added, the median follow-up of patients without recurrence was 75 months for suture repair and 81 months for mesh repair.

\section{Recurrence}

In the 2003 study update, 25 new recurrences were found that had not been present during the original study. The 10 -year cumulative rate of recurrence was $63 \%$ for suture repair and 32\% for prosthetic repair $(P<0.001)$ (Fig. 1). In a univariate analysis, surgery for abdominal aortic aneurysm $(P=0.01)$ and infection $(P=0.02)$ were identified as risk 
TABLE 1. Baseline Characteristics of the Patients With Incisional Hernia, According to Study Group*

\begin{tabular}{|c|c|c|}
\hline Variable & $\begin{array}{l}\text { Suture Repair } \\
\qquad(\mathbf{N}=97)\end{array}$ & $\begin{array}{l}\text { Mesh Repair } \\
(\mathbf{N}=\mathbf{8 4})\end{array}$ \\
\hline Gender, M:F & $1,0: 1$ & $1,5: 1$ \\
\hline \multicolumn{3}{|l|}{ Age, y } \\
\hline Median & 63 & 57 \\
\hline Range & $25-82$ & $23-85$ \\
\hline \multicolumn{3}{|l|}{ Body mass index ${ }^{\dagger}$} \\
\hline Median & 26,0 & 26,2 \\
\hline Range & $20,0-41,5$ & $19,7-41,5$ \\
\hline Prostatism (no. of males) & $6 / 47(13)$ & $1 / 49(1)$ \\
\hline Smoking (\%) & 27/92 (29) & $32 / 82(39)$ \\
\hline Infection $(\%)$ & $2 / 92(2)$ & $3 / 82(4)$ \\
\hline Hematoma (\%) & $8 / 96(8)$ & 9/83 (11) \\
\hline \multicolumn{3}{|l|}{ Intraoperative size of hernia, $\mathrm{cm}^{2}$} \\
\hline Median & 20 & 24 \\
\hline Range & $1-225$ & $1-160$ \\
\hline \multicolumn{3}{|c|}{$\begin{array}{l}\text { Main reason for laparotomy before } \\
\text { repair, No. }{ }^{\S}\end{array}$} \\
\hline Gastrointestinal operation & 48 & 38 \\
\hline Gynecologic operation & 16 & 15 \\
\hline Cholecystectomy & 9 & 5 \\
\hline Abdominal aortic aneurysm & 6 & 12 \\
\hline Other & 28 & 30 \\
\hline
\end{tabular}

*For some variables, data were not available for all the patients in the group.

tThe body mass index was calculated as the weight in kilograms divided by the square of the height in meters.

${ }^{\S}$ Some patients had undergone more than 1 previous laparotomy.

TABLE 2. Rate of Recurrence After Suture or Mesh Repair of Incisional Hernia

\begin{tabular}{lcccc}
\hline Type of Repair & $\begin{array}{c}\text { No. of } \\
\text { Patients }\end{array}$ & $\begin{array}{c}\text { No. of } \\
\text { Recurrences }\end{array}$ & $\begin{array}{c}\text { 10-y } \\
\text { Cumulative } \\
\text { Rate of } \\
\text { Recurrence }\end{array}$ & $\boldsymbol{P}$ Value \\
\hline Suture repair & 97 & 54 & 63 & $P<0.001^{*}$ \\
Mesh repair & 84 & 27 & 32 & \\
Total & 181 & 81 & & \\
\hline & & & $(\%)$ \\
\hline
\end{tabular}

factors for recurrence. Among the preoperative data that were not identified as risk factors for recurrence were age, gender, primary or first recurrent hernia, size of the defect, smoking, prostatism, diabetes, obesity, and steroid use. In a subgroup of 50 patients with small incisional hernia $\left(\leq 10 \mathrm{~cm}^{2}\right)$, the

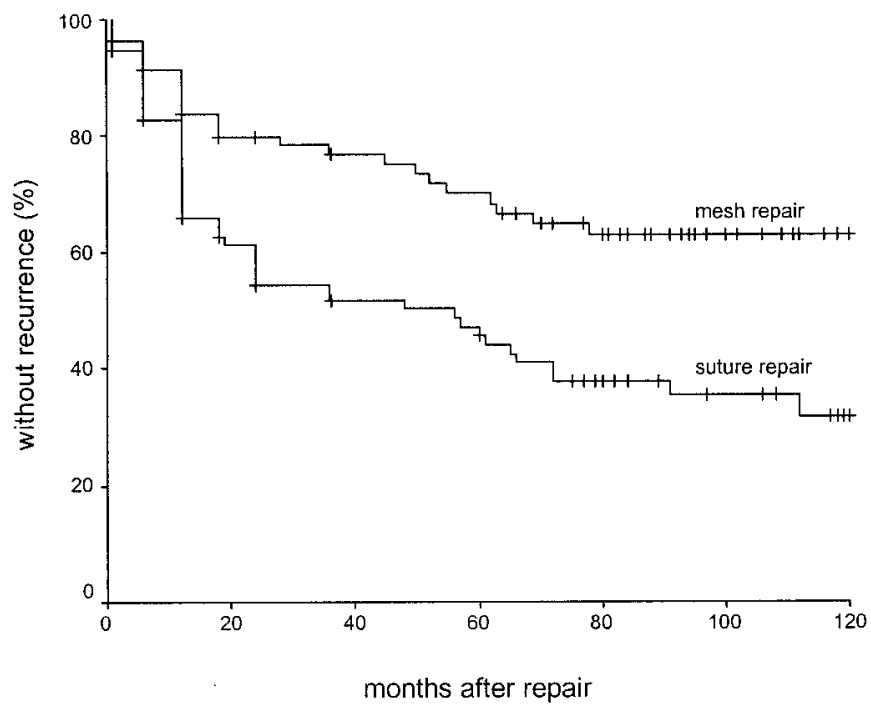

FIGURE 1. Kaplan-Meier curves for recurrence of hernia after repair of a primary or first recurrent incisional hernia according to whether the patient was assigned to suture repair (97 patients) or mesh repair (84 patients). There were significantly fewer recurrences in patients who were assigned to mesh repair $(P<0.001)$.

10-year cumulative rate of recurrence was $67 \%$ after suture repair, compared with $17 \%$ after mesh repair $(P=0.003)$.

\section{Complications}

Long-term follow-up was obtained from 126 patients. In these patients, the median follow-up was 97 months for suture repair and 98 months for mesh repair. Sixty-six patients were in the suture repair group, while 60 patients were in the mesh repair group. In the mesh repair group, 17\% (10 patients) suffered a hernia repair-related complication, compared with $8 \%$ (5 patients) in the suture repair group ( $P=$ $0.17)$. Complications in the mesh repair group were small bowel obstruction (7 patients), fistula from mesh to skin (3 patients), mesh infection (1 patient) and enterocutaneous fistula (2 patients). Complications in the suture repair group were small bowel obstruction ( 3 patients), strangulated hernia (1 patient), and burst abdomen (1 patient). Of the 10 patients in the mesh repair group that had a complication that could be related to the hernia repair, 2 had undergone further surgery before the onset of the complication.

\section{Successive Incisional Hernia Repair}

In the suture repair group, 23 of 66 patients (35\%) went through repair of a recurrence of an incisional hernia repair that was performed in the framework of the trial, while 7 of $60(12 \%)$ of the mesh repair patients underwent a successive hernia repair $(P=0.003)$. 


\section{Pain}

Twenty-seven percent of suture repair patients had experienced scar pain during the last month, compared with $20 \%$ of mesh repair patients $(P=0.53)$. When asked whether they had experienced scar pain during the past years, $23 \%$ of suture repair patients and $20 \%$ of mesh repair patients answered affirmatively $(P=0.83)$. In a visual analogue scale, suture repair patients rated their scar pain during the past month as 1.17 , while patients in the mesh repair group rated the pain as $1.12(P=0.68)$. Patients in the suture repair group rated their scar pain during the last years as 1.30 on average, while patients in the mesh repair group rated the pain as 1.12 $(P=0.75)$.

Thirty-six percent of suture repair patients had experienced abdominal pain during the last month, compared with $20 \%$ of patients with mesh repair $(P=0.05)$. When asked whether they had experienced abdominal pain in the years after incisional hernia repair, 39\% of suture repair patients and $18 \%$ of mesh repair patients answered affirmatively $(P=$ $0.01)$. In a visual analogue scale, suture repair patients rated their abdominal pain during the past month as 1.9 on average, while patients in the mesh repair group rated the pain as 1.0 $(P=0.04)$. Patients in the suture repair group rated their abdominal pain in the last years as 2.2 on average, while patients in the mesh repair group rated the abdominal pain during the past years as $1.0(P=0.009)$.

\section{Cosmetics}

Forty-seven percent of suture repair patients and 52\% of mesh repair patients were satisfied with the way their abdomen looked $(P=0.86)$. Patients were asked to score the cosmetic result on a visual analogue scale, in which 0 meant the worst possible result and 10 the best possible result. Both suture repair and mesh repair patients rated the cosmetic appearance as $6.0(P=0.70)$. Twenty-four percent of suture repair patients and $20 \%$ of mesh repair patients was ashamed of the appearance of their abdomen $(P=0.52)$. Whenever patients answered that their abdomen looked bad, asymmetry of the abdomen was the most frequent objection, which was the same in both groups. Other frequent complaints were a disfiguring scar and bulging, often representing a recurrent hernia.

\section{Satisfaction}

Patients were asked to take into consideration all possible positive and negative effects of the incisional hernia repair and state whether they were satisfied with the procedure or not. In the suture repair group, $64 \%$ was satisfied, while in the mesh repair group, $77 \%$ was satisfied $(P=0.12)$. When patients were asked why they were dissatisfied, most patients answered that they had suffered a recurrence. Other reasons were scar and abdominal pain and a disappointing cosmetic result.

\section{DISCUSSION}

Our study provides evidence that in the long-term mesh repair of incisional hernia is superior to suture repair. Recurrence is more frequent after suture repair, while the incidence of hernia repair-related complications, scar pain, cosmetic result, and patient satisfaction is comparable for both groups. Two findings in particular are new and important. First, the incidence and intensity of abdominal pain are lower after mesh repair than after suture repair. Second, recurrence of incisional hernia continues to occur up to 10 years after hernia repair.

The current study established that the recurrence rate after suture repair of incisional hernia rises to an unacceptable level 10 years after surgery (63\%). Although the results of mesh repair are disappointing as well, its recurrence rate is approximately half of the recurrence rate after suture repair. For small incisional hernias $\left(\leq 10 \mathrm{~cm}^{2}\right)$, the difference was even more apparent. Not only did mesh repair patients suffer fewer recurrences than suture repair patients, they also underwent fewer successive repairs of a recurrence.

Remarkably, recurrence continues up to 10 years after incisional hernia repair, also after mesh repair. It is therefore likely that recurrence rates are generally underestimated, because most studies are either not prospective or do not include long-term follow-up. Our results show that long-term follow-up is mandatory in any study dealing with recurrence of incisional hernia repair.

Comparison of our data with the results of others is troublesome, because of the lack of randomized controlled trials. In 2001, Korenkov et $\mathrm{al}^{25}$ published the results of a randomized controlled trial of incisional hernia repair. Korenkov et $\mathrm{a}^{25}$ concluded that suture repair of incisional hernia was safe and did not result in higher recurrence rates. However, the trial was discontinued due to the severity of mesh infections. In our study, we encountered only few mesh infections (incidence 3.7\%), and the course of these infections was mild. In 2001, Arroyo et $\mathrm{al}^{28}$ published a randomized controlled trail on umbilical hernia repair. Although umbilical hernia may differ from incisional hernia etiologically, treatment modalities for ventral hernia repair are similar and results may therefore be compared. In line with our results, Arroyo et $\mathrm{al}^{28}$ reported that even for small umbilical hernias, mesh repair results in significantly fewer recurrences than suture repair.

Mesh repair of incisional hernia has been associated with complications, such as enterocutaneous fistula and small bowel obstruction. In our study, we found no significant difference in the incidence of complications. In a study by Leber et $\mathrm{al},{ }^{29}$ the incidence of small bowel obstruction following mesh repair of incisional hernia was $5.4 \%$, which compares well to our $11.7 \%$. The incidence of enterocutaneous fistula following mesh repair of incisional hernia is 
thought to be low. ${ }^{30}$ Leber et al, ${ }^{29}$ reported a 3.5\% incidence of enterocutaneous fistula and a $5.9 \%$ incidence of mesh to skin fistulas. In the current study, $5 \%$ of patients developed a fistula from mesh to skin (sinus tract), while 3\% developed an enterocutaneous fistula. Although numbers were too small to reach significance, we believe that the importance of this finding is determined by the severity of the complication. On the other hand, others have reported the intra-abdominal use of meshes to be safe. ${ }^{31}$ Moreover, the occurrence of burst abdomen and strangulated hernia in the suture repair group may equal the enterocutaneous fistulas in complication severity.

Because physical complaints are an important reason for performing incisional hernia repair, any analysis of incisional hernia repair should include discomfort. Others have suggested that up to $50 \%$ of patients having undergone mesh repair of an incisional hernia developed complaints because of a reduced mobility of the abdominal wall. ${ }^{20,32}$ Our study does not reveal any difference in scar or superficial pain between mesh and suture repair patients. Moreover, abdominal pain was less frequent (18\% versus 39\%) and less intense in patients having undergone mesh repair. We think that discomfort following incisional hernia repair is caused by tension on the abdominal wall and that the relative decrease in pain after mesh repair may be caused by the tension-free technique that is applied in mesh repair, but not in suture repair.

Cosmetics too may play a key role in the patient's wish to have an incisional hernia repaired. Remarkably, in our study, only $47 \%$ to $52 \%$ of patients considered the cosmetic result satisfactory. It is important for surgeons to be aware of this general discontent among incisional hernia repair patients. Adequate information preoperatively may result in some patients refraining from incisional hernia repair.

In conclusion, our study is the first and only to provide prospective long-term follow-up of incisional hernia repair. It proves that mesh repair is superior to suture repair for both small and large incisional hernias. Mesh repair results in lower recurrence rates and less discomfort in the long term, while mesh repair is not associated with an increased incidence of complications. We conclude that to reduce the morbidity and the costs associated with incisional hernia repair and to prevent patients from undergoing pointless surgery, suture repair of incisional hernia should be completely abandoned.

\section{REFERENCES}

1. Mudge M, Hughes LE. Incisional hernia: a 10-year prospective study of incidence and attitudes. Br J Surg. 1985;72:70-71.

2. Lewis RT, Wiegand FM. Natural history of vertical abdominal parietal closure: Prolene versus Dexon. Can J Surg. 1989;32:196-200.

3. Sugerman HJ, Kellum JM Jr, Reines HD, et al. Greater risk of incisional hernia with morbidly obese than steroid-dependent patients and low recurrence with prefascial polypropylene mesh. Am J Surg. 1996;171: $80-84$.

4. Hodgson NC, Malthaner RA, Ostbye T. The search for an ideal method of abdominal fascial closure: a meta-analysis. Ann Surg. 2000;231:436442.

5. Hoer J, Lawong G, Klinge U, et al. [Factors influencing the development of incisional hernia: a retrospective study of 2,983 laparotomy patients over a period of 10 years]. Chirurg. 2002;73:474-480.

6. National Center for Health Statistics. Combined surgery data (NHDS and NSAS) data highlights. Available at: http://www.cdc.gov/nchs/ about/major/hdasd/combtab.htm. 1996.

7. Franz MG, Kuhn MA, Nguyen K, et al. Transforming growth factor beta(2) lowers the incidence of incisional hernias. J Surg Res. 2001;97: 109-116.

8. Prismant. Utrecht, the Netherlands: National Medical Registration; 2002.

9. DeMaria EJ, Moss JM, Sugerman HJ. Laparoscopic intraperitoneal polytetrafluoroethylene (PTFE) prosthetic patch repair of ventral hernia: prospective comparison to open prefascial polypropylene mesh repair. Surg Endosc. 2000;14:326-329.

10. Read RC, Yoder G. Recent trends in the management of incisional herniation. Arch Surg. 1989;124:485-488.

11. Manninen MJ, Lavonius M, Perhoniemi VJ. Results of incisional hernia repair: a retrospective study of 172 unselected hernioplasties. Eur J Surg. 1991;157:29-31.

12. Paul A, Korenkov M, Peters S, et al. Unacceptable results of the Mayo procedure for repair of abdominal incisional hernias. Eur J Surg. 1998;164:361-367.

13. Anthony T, Bergen PC, Kim LT, et al. Factors affecting recurrence following incisional herniorrhaphy. World J Surg. 2000;24:95-100.

14. Luijendijk RW, Hop WC, van den Tol MP, et al. A comparison of suture repair with mesh repair for incisional hernia. $N$ Engl J Med. 2000;343: 392-398.

15. Korenkov M, Sauerland S, Arndt M, et al. Randomized clinical trial of suture repair, polypropylene mesh or autodermal hernioplasty for incisional hernia. Br J Surg. 2002;89:50-56.

16. Luijendijk RW, Lemmen MH, Hop WC, et al. Incisional hernia recurrence following "vest-over-pants" or vertical Mayo repair of primary hernias of the midline. World J Surg 1997;21:62-65.

17. van der Linden FT, van Vroonhoven TJ. Long-term results after surgical correction of incisional hernia. Neth J Surg. 1988;40:127-129.

18. Liakakos T, Karanikas I, Panagiotidis H, et al. Use of Marlex mesh in the repair of recurrent incisional hernia. Br J Surg. 1994;81:248-249.

19. Langer I, Herzog U, Schuppisser JP, et al. [Preperitoneal prosthesis implantation in surgical management of recurrent inguinal hernia: retrospective evaluation of our results 1989-1994] Die praperitoneale Protheseneinlage zur operativen Versorgung der Rezidivleistenhernie. Retrospektive Beurteilung unserer Resultate von 1989-1994. Chirurg. 1996;67:394-402.

20. McLanahan D, King LT, Weems C, et al. Retrorectus prosthetic mesh repair of midline abdominal hernia. Am J Surg. 1997;173:445-449.

21. Park A, Birch DW, Lovrics P. Laparoscopic and open incisional hernia repair: a comparison study. Surgery. 1998;124:816-821.

22. Schumpelick V, Klosterhalfen B, Muller M, et al. [Minimized polypropylene mesh for preperitoneal net plasty (PNP) of incisional hernias] Minimierte Polypropylen-Netze zur praperitonealen Netzplastik (PNP) der Narbenhernie. Eine prospektive randomisierte klinische Studie. Chirurg. 1999;70:422-430.

23. Toniato A, Pagetta C, Bernante $\mathrm{P}$, et al. Incisional hernia treatment with progressive pneumoperitoneum and retromuscular prosthetic hernioplasty. Langenbecks Arch Surg. 2002;387:246-248.

24. Kingsnorth A, LeBlanc K. Hernias: inguinal and incisional. Lancet. 2003;362:1561-1571.

25. Korenkov M, Paul A, Sauerland S, et al. Classification and surgical treatment of incisional hernia: results of an experts' meeting. Langenbecks Arch Surg. 2001;386:65-73.

26. Paul A, Lefering R, Kohler L, et al. [Current practice of incisional hernia reconstruction in Germany] Gegenwartige Praxis der Narbenhernienrekonstruktion in der Bundesrepublik Deutschland. Zentralbl Chir. 1997; 122:859-861.

27. Flum DR, Horvath K, Koepsell T. Have outcomes of incisional hernia repair improved with time? A population-based analysis. Ann Surg. 2003;237:129-135. 
28. Arroyo A, Garcia P, Perez F, et al. Randomized clinical trial comparing suture and mesh repair of umbilical hernia in adults. $\mathrm{Br} J$ Surg. 2001;88:1321-1323.

29. Leber GE, Garb JL, Alexander AI, et al. Long-term complications associated with prosthetic repair of incisional hernias. Arch Surg. 1998; 133:378-382.

30. Morris-Stiff GJ, Hughes LE. The outcomes of nonabsorbable mesh placed within the abdominal cavity: literature review and clinical experience. J Am Coll Surg. 1998;186:352-367.

31. Vrijland WW, Jeekel J, Steyerberg EW, et al. Intraperitoneal polypropylene mesh repair of incisional hernia is not associated with enterocutaneous fistula. Br J Surg. 2000;87:348-352.

32. Welty G, Klinge U, Klosterhalfen B, et al. Functional impairment and complaints following incisional hernia repair with different polypropylene meshes. Hernia. 2001;5:142-147.

\section{Discussions}

Dr. Theodore N. Pappas (Durham, North Carolina): I think this is an important paper because it deals with a common disease. We are not talking about subspecialty care here, we are talking about something that impacts on almost every abdominal surgeon in the country.

The paper undoubtedly will be criticized - as most multicenter trials are-with respect to whether there was adequate standardization of the operation. There will be talk about the drop-out rate, the large number of patients who died, and the cross-over. But taking those criticisms into account, I think the data is still very compelling, because the differences in recurrence between mesh and suture repair are large, the differences are obvious, and, most importantly, there is a clinically important difference between the 2 groups. I have several questions.

Number one, it looked as though to me your recurrence rates dominate your other outcomes, which are satisfaction, pain, cosmetics. It is undoubtedly true that if a patient has a recurrence, that fact will impact on the other 3 endpoints. So did you do an analysis of the non-recurrent patients to see if in fact there were differences in those 3 endpoints? That might tell you more about satisfaction, pain, and cosmetics and not allow the recurrence to bias those endpoints.

You mention in your manuscript, although you didn't mention it today, that you used Vicryl mesh behind your prosthetic mesh in an effort to protect the bowel and avoid fistulization. Is there compelling data that we should be doing this? Do you have enough patients that you have analyzed that have allowed you to say convincingly that Vicryl mesh does protect from fistulization?

Could you tell us a little bit more about the relationship between size and recurrence? You did some analysis, but is there a linear relationship between size and recurrence? There are many people who believe that the size issue is in fact more important or at least as important as the type of repair.

Finally, do you have any experience with component separation? Component separation is a very commonly used technique, which allows to you avoid mesh but yields a tension-free repair.

Dr. J. W. A. Burger (Rotterdam, The Netherlands): The first question, did we look at patient satisfaction, abdominal pain and scar pain in patients that did not have a recurrence? We did not look at that specifically. We did, however, see that what you just hypothesized is correct. Patient satisfaction and abdominal pain are strongly related to incisional hernia recurrence. I can't tell you how it was in patients that did not suffer a recurrence.

The second question about the use of a Vicryl mesh between the polypropylene mesh and the bowels. We actually did not do that in many patients. We only did it if we could not close the peritoneum and if that was not possible, if we could not position the omentum between mesh and bowels. At that point in 1991, we thought that Vicryl might prevent adhesion formation. By now we know from experimental studies that this is not the case and therefore, we are not using Vicryl for that purpose any more.

The third question was, do size and recurrence relate? According to our data, they do not; that is, not for suture repair. We looked at different size groups and studied whether the recurrence rate was dependent on the size of the hernia, and it was not. As I have shown earlier, we found that the recurrence rates of small incisional hernias, smaller than $10 \mathrm{~cm}^{2}$, are still very high.

The fourth question was about the Ramirez component separation technique. Yes, we are looking into the Ramirez technique and mesh repair. At this moment, we are participating in a Dutch trial that will establish whether the use of a mesh should be incorporated in the Ramirez technique for additional strengthening of the abdominal wall. The Ramirez technique without mesh offers better results than just plain suture repair, but recurrence rates are still quite high.

Dr. Charles E. Lucas (Detroit, Michigan): No paper at this meeting highlights our total ignorance about the physiology of wound healing than does this paper. As world surgeons, we really have to look at the cause for this terrible problem, rather than discarding suture repair. We have to think of a physiologic solution and not a mechanical solution.

We know that smoking interferes with wound healing. Do you have any comparison between nicotine use in your 2 groups? Is there any investigation being done by your unit into the amount of collagen or elastin in the tissues of the patients that have recurrence versus those that don't have recurrence?

Dr. J. W. A. Burger (Rotterdam, The Netherlands): The first question was about smoking. In the current study we did not find that smoking was a risk factor for incisional hernia recurrence. We do, however, believe that it is. 
Regarding your second question on collagen and elastin, we are definitely looking into that both experimentally and clinically. We are trying to identify patients who may have a connective tissue disorder associated with decreased collagen and elastin amounts. However, for obvious reasons, it was not possible to incorporate this in this update of this clinical trial.

Dr. Carlos A. Pellegrini (Seattle, Washington): Presumably this series included mostly patients repaired by the open technique and a lot of the recurrences seen in the mesh patients may have been due to infectious problems of the wound. In these days, as you know, many hernias like this, less than 6 centimeters, are treated with laparoscopic technique, which doesn't involve opening any wounds. Do you have any comments on the role of laparoscopy and tensionfree repair with mesh repair may do to these results?

Dr. J. W. A. Burger (Rotterdam, The Netherlands): All of our patients were operated on by the open technique. Regarding laparoscopy, to this moment, there are no large randomized trials proving that laparoscopy is either better or worse than the open technique. I personally feel that laparoscopy could be beneficial because the abdominal wall adjacent to the defect, where wound healing is bound to be poor, is left alone. However, there is no proof of that yet.

Dr. David W. Easter (San Diego, California): I actually like mesh. But I wonder if you have proven your point. I note you use 1 centimeter bites for your fascial closure with suture and at least a 2 centimeter overlap for your mesh. Haven't you proven that 1 centimeter bites are ineffective and that mesh doesn't matter?

Dr. J. W. A. Burger (Rotterdam, The Netherlands): Regarding the suture technique and how we placed the sutures at 1 centimeter intervals and at least 1 centimeter from the wound edge: we applied the rules of Jenkins and Israelsson, who reported that we have to suture with a suture to wound length ratio of at least 4 to 1 . That is how we did it.

Regarding the overlap of the mesh. Two centimeters may actually be too little. We think we may have lower recurrence rates if we increase the overlap of the mesh, and nowadays we do.

Dr. Josef E. Fischer (Boston, Massachusetts): Your conclusions are really recurrence based. But you also have a difference in complications, which perhaps if you had 150 more patients in each group and with the same rate of complications that would have been a statistically significant difference.

Now, if you look at the difference of complications, as you well know, some of them are pretty serious complica- tions, such as mesh erosion in the bowel, which is getting to be an increasing problem, which I see a fair number of those patients. Have you looked at the cost, the long-term cost of the differences between the two groups? Because it doesn't take too many mesh bowel fistulas to give a very substantial difference in outcome if one is looking at cost.

Dr. J. W. A. Burger (Rotterdam, The Netherlands): Regarding your first question about the number of complications, the trial was not meant to look at complications as a primary endpoint, so, unfortunately, the number of patients may be too low to prove significant differences. We will never know until a new trial, which studies complications prospectively, is presented.

Regarding the severity of the complications and the costs, we have not looked at the cost specifically, which is quite difficult retrospectively. Enterocutaneous fistulas are severe complications, but so were the burst abdomen and the strangulated hernia in the suture repair group. Furthermore, we looked at the number of reoperations in both groups, and we found that only $12 \%$ of mesh repair patients had undergone subsequent hernia repair, compared to $35 \%$ of suture repair patients. So costs are made here as well. Moreover, most of the suture repair patients got a mesh repair the second time.

Dr. Stephen J. Mathes (San Francisco, California): I appreciate your paper and especially your long-term followup. My question relates to several areas.

One question relates to the geography of the incisional hernia and recurrence rates. Were more of those in the midline or were some of those recurrences occurring laterally or in proximity to the inguinal region? In those instances, can you describe how you handled the repair where there was no fascia available? For instance, did you attach into bone or into the costal margin?

Second, in our review of our experience to this group several years ago, we found that there was a difference in how you had to manage patients depending on their skin stability or skin coverage over the hernia. And I wondered if you might comment: Was there a group of patients who didn't have stable skin or presented with radiated abdominal walls? Did that make a difference in your recurrence rate whether you used mesh or the suture technique?

Dr. J. W. A. Burger (Rotterdam, The Netherlands): Regarding location of the hernia, they were all midline incisional hernias, and recurrence was defined as a recurrence within 7 centimeters of the original location of the hernia. We did not encounter problems with fascia in the pubic region or in the costal region and I cannot answer the question how we would have done it had it occurred. We did not encounter 
skin problem either. I think this is because we included small incisional hernias, smaller than 6 centimeters in diameter.

Dr. Lawrence W. Way (San Francisco, California): My question is really very similar to Dr. Fischer's question. But leaving cost aside, it seems to me that you need to take into account all of your outcomes and not focus entirely or so heavily on the recurrence rate, because these enterocutaneous fistulas are really a major problem and they don't seem to be as common in the suture closures. We have a steady stream of these patients. And the morbidity is really huge.

And the ultimate question is, how many recurrences equals one enterocutaneous fistula? You can subjectively or in some way or another weight these various outcomes and recalculate your feelings about the 2 operations. I would say that another cutaneous fistula with infection and all the terrible difficulty in fixing it equals about 10 recurrences, in my view. If you have a sprinkling of those throughout, you really have to think about it.

Dr. J. W. A. Burger (Rotterdam, The Netherlands): What I can say about that is that I know of the possible effects of enterocutaneous fistulas. In our 2 patients, the course was not so dramatic. However, if we are talking about one enterocutaneous fistula equating many recurrences, taking recurrence for granted, we should keep in mind that an operation which results in almost $70 \%$ recurrence is not a good operation, whatever the alternative may be. If you do not want to perform mesh repair, that does not necessarily mean you should perform suture repair, because we now know that recurrence rates are incredibly high.

Dr. Haile T. Debas (San Francisco, California): Dr. Burger, this is one comment you don't have to answer. I just rise to make a short and obvious comment on the 4 papers presented this morning. They are all double-blind prospective clinical trials. As such, this morning's papers present something of a watershed. We have arrived in the era of evidencebased surgical practice. And I want to congratulate the committee.

Dr. R. Scott Jones (Charlottesville, Virginia): I would like to make a comment about this presentation and discussion, and I want to extend congratulations to the presenter, Dr. Burger, who did the best presentation I think I have ever heard and he handled the questions from a tough bunch of people better than I could possible have imagined. And you have really made my day. Thank you. 